\title{
Performance investigation of quasi-Newton-based parallel nonlinear FEM for large-deformation elastic-plastic analysis over 100 thousand degrees of freedom
}

\author{
Yasunori YUSA*, Shota MIYAUCHI ${ }^{* *}$ and Hiroshi OKADA*** \\ * Department of Mechanical and Intelligent Systems Engineering, Graduate School of Informatics and Engineering, \\ The University of Electro-Communications \\ 1-5-1 Chofugaoka, Chofu, Tokyo 182-8585, Japan \\ E-mail: y.yusa@uec.ac.jp \\ ** Department of Mechanical Engineering, Graduate School of Science and Technology, Tokyo University of Science \\ ${ }^{* * *}$ Department of Mechanical Engineering, Faculty of Science and Technology, Tokyo University of Science \\ 2641 Yamazaki, Noda, Chiba 278-8510, Japan
}

Received: 9 February 2021; Revised: 29 March 2021; Accepted: 7 May 2021

\begin{abstract}
Quasi-Newton-based nonlinear finite element methods were extensively studied in the 1970s and 1980s. However, they have almost disappeared due to their poorer convergence performance than the Newton-Raphson method. An advantage of quasi-Newton methods over the Newton-Raphson method is shorter computational time even with a larger number of iterations. The speedup must grow as the number of degrees of freedom (DOFs) increases. Since computers and computational methods have been advancing steadily in the last 40 years, significant speedup can be expected at present. Therefore, we present a framework of a quasi-Newton-based parallel nonlinear finite element method consisting of a quasi-Newton method, implementation for parallel computing and a nonlinear material model. The advances of the present framework are a large number of DOFs and the use of a modern nonlinear material model. The number of DOFs exceeded 100 thousand in all analyses and reached one million in some analyses. This was enabled by the implementation of a quasi-Newton method for parallel computing and the use of a parallel sparse direct solver library. Note that, for more than several or ten million DOFs, an iterative linear solution method is generally preferred, resulting in the loss of the advantage of quasi-Newton methods. Furthermore, a modern finite-strain elastoplasticity material model with a realistic multilinear stress-strain curve was used in the present study, whereas an infinitesimal elastoplasticity model with a bilinear stress-strain curve was popular in the 1980s. Performance investigation of the present framework is given in the present paper. The quasi-Newton-based present framework achieved speedup of more than 30 times in modern large-deformation elastic-plastic analyses involving approximately one million DOFs, compared to the Newton-Raphson method.
\end{abstract}

Keywords : Nonlinear finite element method, Large-deformation elastic-plastic analysis, Crack problem, QuasiNewton method, Parallel computing, Speedup

\section{Introduction}

In the 2010s, finite element analysis of strongly nonlinear problems such as a large-deformation elastic-plastic problem became very popular in practical situations. In addition, finite element analysis of problems having more than 100 thousand degrees of freedom (DOFs) became common in the 2000s and 2010s, due to the advances in computers and the spread of parallel sparse direct solver libraries such as MUMPS, PARDISO, SPOOLES, SuperLU, UMFPACK and WSMP in alphabetical order. For example, Arai et al. (2018) analyzed cyclic elastic-plastic problems of a cracked specimen. The number of DOFs of the finite element analysis model was 370 thousand. Okada et al. (2020) solved largedeformation elastic-plastic problems with a crack. The number of DOFs was 970 thousand. Such finite element analyses generally require very long computational time. Furthermore, multiple analysis cases should usually be computed in a 
design work to find an appropriate design, as well as in an accident investigation to discover the mechanical phenomena of the accident. Such situations would lead to considerably long computational time in total.

The reason why nonlinear finite element analysis for more than 100 thousand DOFs requires long computational time can be found in solution procedures for linear systems of equations. For problems with less than one million DOFs, a direct linear solution method, such as the LU (lower-upper), LL (lower-lower) and LDL (lower-diagonal-lower) matrix factorization methods, is preferred in general. A parallel sparse direct solver library is usually used in the program. Note that the use of an iterative linear solution method must exhibit shorter computational time for more than several or ten million DOFs, compared to the direct linear solution method. Such problems are out of scope of the present study. Since a matrix factorization of the direct method requires time complexity that is a high-degree polynomial of the number of DOFs, its computational time grows steeply as the number of DOFs increases. The time complexity of the matrix factorization is typically $O\left(n^{3}\right)$ for a dense matrix and $O\left(n^{\frac{7}{3}}\right)$ for a band matrix, where $n$ is the number of DOFs. That in a sparse direct solver depends on its methodology. Furthermore, nonlinear finite element analysis requires a large number of solution procedures for linear systems of equations due to incremental steps in conjunction with NewtonRaphson iteration steps. For this problem, other nonlinear solution methods such as the modified Newton method and quasi-Newton methods were extensively studied in the 1970s and 1980s, namely, Nayak and Zienkiewicz (1972), Crisfield (1979), Matthies and Strang (1979), Bathe and Cimento (1980), Geradin et al. (1980, 1981), Wissmann and Hauck (1983), and Gelin and Picart (1988). Referring to these studies, some books such as Hisada and Noguchi (1995), Wriggers (2008), and de Borst et al. (2012) stated that a quasi-Newton method can achieve shorter computational time than the NewtonRaphson method, even though it requires a larger number of iterations. However, to the best of the authors' knowledge, nowadays, quasi-Newton methods are rarely used for practical nonlinear finite element analysis. According to de Borst et al. (2012), poor convergence performance seems to have decreased the popularity of quasi-Newton methods. Note that some researchers nevertheless used quasi-Newton methods for fast nonlinear finite element analysis of engineering problems, such as contact (Laursen and Maker, 1995), strip rolling (Mei et al., 2012), and soils and foundations (Shigeno, 2013).

The speedup of a quasi-Newton method over the Newton-Raphson method must grow as the number of DOFs increases. This is because a quasi-Newton method is able to reduce the frequency of matrix factorization, although the frequency of forward and backward substitutions increases. A matrix factorization requires time complexity that is a higher-degree polynomial of the number of DOFs, than forward and backward substitutions. For a dense matrix, the time complexity of the matrix factorization is $O\left(n^{3}\right)$, whereas that of the forward and backward substitutions is $O\left(n^{2}\right)$. For a band matrix, they are $O\left(n^{\frac{7}{3}}\right)$ and $O\left(n^{\frac{5}{3}}\right)$, respectively. Furthermore, the number of DOFs that can be solved has become much larger in the 2010 s compared to those in the 1980 s, due to the advances in computers and computational methods. Hence, significant speedup can be expected at present. This is why we reinvestigated the computational performance of a quasi-Newton-based nonlinear finite element method. The authors believe that a quasi-Newton-based nonlinear finite element method would be a good alternative to the Newton-Raphson method for problems with a large number of DOFs. Here, we have two major questions, i.e., the convergence performance and the speedup of quasi-Newton methods in modern nonlinear finite element analysis. They were investigated in the present study.

In the present paper, we present a framework of a quasi-Newton-based parallel nonlinear finite element method consisting of a quasi-Newton method, implementation for distributed-memory parallel computer environments and a modern finite-strain elastoplasticity material model. A quasi-Newton method consists of a solution procedure for a linear system of equations and vector operations such as vector additions and dot products. In the present framework, the linear system of equations is solved by a parallel sparse direct solver library. The vector operations were coded directly in the program. Then, computational performance of the present framework was investigated. The convergence performance and the speedup of large-deformation elastic-plastic analysis for 100 thousand DOFs were investigated in detail through a crack problem. In this analysis, a modern finite-strain elastoplasticity model with a realistic multilinear stress-strain curve was used, whereas infinitesimal elastoplasticity model with a bilinear stress-strain curve was popular in the 1980s. Note that we are not aiming to analyze severe problems such as plastic instability and bifurcation. Moreover, a weak scaling test was carried out for investigating the change of the convergence performance and the speedup for different numbers of DOFs. The number of DOFs ranged from 100 thousand to approximately 800 thousand in the week scaling test. Finally, we analyzed three examples to confirm the convergence performance and the speedup. Each of the three examples had approximately one million DOFs. The numerical results in the present paper demonstrated that the quasiNewton-based present framework is able to achieve significant speedup for more than 100 thousand DOFs, compared to the Newton-Raphson method. 


\section{Present framework of quasi-Newton-based nonlinear finite element method for large-deformation elastic-plastic analysis on distributed-memory parallel computer environments}

In this section, first, a nonlinear system of equations is derived, followed by solution methods including a quasiNewton method. Then, we present the algorithm and the implementation for distributed-memory parallel computer environments. Finally, a finite-strain elastoplasticity model that was used in the present framework is briefly explained.

\subsection{Nonlinear finite element method based on a quasi-Newton method}

In this subsection, a nonlinear system of equations is derived from the principle of virtual work. Three solution methods for this nonlinear system of equations are presented, namely, the Newton-Raphson method, the modified Newton method and a quasi-Newton method.

A boundary value problem is defined in Fig. 1. The domain of the body is represented by $\Omega$. The boundary of $\Omega$ is $\Gamma$. Then, let $\Gamma_{u}$ and $\Gamma_{t}$ be the displacement-prescribed boundary and the traction-prescribed boundary, respectively. They satisfy both $\Gamma_{u} \cup \Gamma_{t}=\Gamma$ and $\Gamma_{u} \cap \Gamma_{t}=\emptyset$.

Then, the principle of virtual work in an updated Lagrangian sense is introduced as

$$
\int_{\Omega} \delta \tilde{\boldsymbol{\varepsilon}}^{\mathrm{T}} \boldsymbol{\sigma} \mathrm{d} \Omega-\int_{\Gamma_{t}} \delta \tilde{\boldsymbol{u}}^{\mathrm{T}} \boldsymbol{t} \mathrm{d} \Gamma-\int_{\Omega} \delta \tilde{\boldsymbol{u}}^{\mathrm{T}} \boldsymbol{b} \mathrm{d} \Omega=0,
$$

where $\sigma, \boldsymbol{t}$ and $\boldsymbol{b}$ are the Cauchy stress, the traction prescribed on $\Gamma_{t}$ and the body force prescribed in $\Omega$, respectively. In addition, $\delta \tilde{\boldsymbol{u}}$ is the virtual displacement. $\delta \tilde{\boldsymbol{\varepsilon}}$ is equal to $\frac{1}{2}\left(\partial \delta \tilde{\boldsymbol{u}} / \partial \boldsymbol{x}+(\partial \delta \tilde{\boldsymbol{u}} / \partial \boldsymbol{x})^{\mathrm{T}}\right)$. The virtual displacement, $\delta \tilde{\boldsymbol{u}}$, can be discretized by

$$
\delta \tilde{\boldsymbol{u}} \approx \boldsymbol{N} \delta \boldsymbol{u}
$$

where $\boldsymbol{N}$ and $\delta \boldsymbol{u}$ denote the shape functions and the nodal virtual displacement, respectively. Inserting Eq. (2) into Eq. (1) yields

$$
\boldsymbol{r}=\mathbf{0}
$$

where

$$
\begin{aligned}
& \boldsymbol{r}=\boldsymbol{f}^{\mathrm{int}}-\boldsymbol{f}^{\mathrm{ext}}, \\
& \boldsymbol{f}^{\mathrm{int}}=\int_{\Omega} \boldsymbol{B}^{\mathrm{T}} \boldsymbol{\sigma} \mathrm{d} \Omega, \\
& \boldsymbol{f}^{\mathrm{ext}}=\int_{\Gamma_{t}} \boldsymbol{N}^{\mathrm{T}} \boldsymbol{t} \mathrm{d} \Gamma+\int_{\Omega} \boldsymbol{N}^{\mathrm{T}} \boldsymbol{b} \mathrm{d} \Omega .
\end{aligned}
$$

Here, $\boldsymbol{r}, \boldsymbol{f}^{\text {int }}$ and $\boldsymbol{f}^{\text {ext }}$ denote the residual vector, the internal force vector and the external force vector, respectively. Moreover, $\boldsymbol{B}$ in Eq. (5) is the strain-nodal-displacement matrix, which is equal to $\frac{1}{2}\left(\partial \boldsymbol{N} / \partial \boldsymbol{x}+(\partial \boldsymbol{N} / \partial \boldsymbol{x})^{\mathrm{T}}\right)$. Equation $(3)$ is the nonlinear system of equations to be solved.

This nonlinear system of equations is solved by a nonlinear solution method along with the incremental method. In the incremental method, boundary conditions are set to increase gradually for steps in the time direction. At each incremental step, the nodal displacement, $\boldsymbol{u}$, which is the solution of Eq. (3), is updated by

$$
\boldsymbol{u}^{(k+1)}=\boldsymbol{u}^{(k)}+\Delta \boldsymbol{u}^{(k)}
$$

where $k$ and $\Delta \boldsymbol{u}$ are the incremental step and the nodal displacement increment, respectively. The nodal displacement increment, $\Delta \boldsymbol{u}$, is

$$
\begin{aligned}
& \Delta \boldsymbol{u}^{(k, i+1)}=\Delta \boldsymbol{u}^{(k, i)}+\boldsymbol{d}^{(i)}, \\
& \boldsymbol{d}^{(i)}=-\boldsymbol{K}^{(i)^{-1}} \boldsymbol{r}^{(i)},
\end{aligned}
$$

where $i$ and $\boldsymbol{K}^{(i)}$ are the iteration step and the Jacobian matrix. If we use the Newton-Raphson method as a nonlinear solution method, $\boldsymbol{K}^{(i)}$ is the exact Jacobian matrix:

$$
\boldsymbol{K}^{(i)}=\left(\frac{\partial \boldsymbol{r}}{\partial \boldsymbol{u}}\right)^{(i)}
$$


If the modified Newton method is adopted, $\boldsymbol{K}^{(i)}$ is replaced by the stiffness matrix of an elastic body. Finally, if a quasiNewton method is selected, the Jacobian matrix is approximated by a formula. Although a variety of quasi-Newton methods have been proposed, we adopted the limited-memory version (Nocedal, 1980) of the BFGS (Broyden-FletcherGoldfarb-Shanno) method in the present framework. This method is commonly used in the field of unconstrained optimization. The inverse of the approximate Jacobian matrix of the limited-memory BFGS method is

$$
\begin{aligned}
& \boldsymbol{K}^{(i)^{-1}}=\boldsymbol{V}^{(i-1)^{\mathrm{T}}} \boldsymbol{V}^{(i-2)^{\mathrm{T}}} \cdots \boldsymbol{V}^{(0)^{\mathrm{T}}} \boldsymbol{K}^{(0)^{-1}} \boldsymbol{V}^{(0)} \boldsymbol{V}^{(1)} \cdots \boldsymbol{V}^{(i-1)} \\
& +\boldsymbol{V}^{(i-1)^{\mathrm{T}}} \boldsymbol{V}^{(i-2)^{\mathrm{T}}} \cdots \boldsymbol{V}^{(1)^{\mathrm{T}}} \frac{\boldsymbol{d}^{(0)} \boldsymbol{d}^{(0)} \mathrm{T}}{\boldsymbol{d}^{(0)}{ }^{\mathrm{T}} \boldsymbol{y}^{(0)}} \boldsymbol{V}^{(1)} \boldsymbol{V}^{(2)} \cdots \boldsymbol{V}^{(i-1)} \\
& +\cdots+\boldsymbol{V}^{(i-1)^{\mathrm{T}}} \frac{\boldsymbol{d}^{(i-2)} \boldsymbol{d}^{(i-2)^{\mathrm{T}}}}{\boldsymbol{d}^{(i-2)^{\mathrm{T}}} \boldsymbol{y}^{(i-2)}} \boldsymbol{V}^{(i-1)} \\
& +\frac{\boldsymbol{d}^{(i-1)} \boldsymbol{d}^{(i-1)^{\mathrm{T}}}}{\boldsymbol{d}^{(i-1)^{\mathrm{T}}} \boldsymbol{y}^{(i-1)}}
\end{aligned}
$$

where

$$
\begin{aligned}
& \boldsymbol{y}^{(i-1)}=\boldsymbol{r}^{(i)}-\boldsymbol{r}^{(i-1)}, \\
& \boldsymbol{V}^{(i-1)}=\boldsymbol{I}-\frac{\boldsymbol{y}^{(i-1)} \boldsymbol{d}^{(i-1)^{\mathrm{T}}}}{\boldsymbol{d}^{(i-1)^{\mathrm{T}}} \boldsymbol{y}^{(i-1)}} .
\end{aligned}
$$

Here, $\boldsymbol{I}$ denotes the identity matrix. Moreover, $\boldsymbol{K}^{(0)}$ in Eq. (11) is the initial approximate Jacobian matrix. Although $\boldsymbol{K}^{(0)}$ can be an arbitrary matrix as long as it is invertible, the selection of $\boldsymbol{K}^{(0)}$ highly affects convergence and computational time. We used the stiffness matrix of an infinitesimal elastic body for $\boldsymbol{K}^{(0)}$ in the present framework. This matrix remains constant throughout the incremental and iteration steps. Equation (11) includes a matrix inverse of $\boldsymbol{K}^{(0)^{-1}}$ and many cross products such as $\boldsymbol{y}^{(i-1)} \boldsymbol{d}^{(i-1)^{\mathrm{T}}}$ and $\boldsymbol{d}^{(i-1)} \boldsymbol{d}^{(i-1)^{\mathrm{T}}}$. For the computation of Eq. (9), the multiplication of $\boldsymbol{K}^{(0)^{-1}}$ and a vector is a solution of a linear system of equations for which the coefficient matrix is $\boldsymbol{K}^{(0)}$. This linear system of equations is solved by a parallel sparse direct solver library in the present framework. Then, the multiplication of a cross product and a vector is transformed into the combination of a dot product and a scalar multiplication.

\subsection{Algorithm and implementation for distributed-memory parallel computer environments}

Since parallel computers were not standard in the 1980s, we explain the algorithm and the implementation of the present framework for distributed-memory parallel computer environments. Most parallel sparse direct solver libraries currently support distributed-memory parallel computing. Note that the present framework can also be applied to sharedmemory parallel computers.

Flowcharts of the Newton-Raphson method and the limited-memory BFGS method are shown in Figs. 2 and 3, respectively. A flowchart of the modified Newton method is omitted because it is very similar to that of the NewtonRaphson method. The only difference is the position of the matrix factorization, which is outside the Newton-Raphson iteration. In both Figs. 2 and 3, the algorithm consists of nested loops of incremental steps and iteration. In the inner loop of the iteration, $\boldsymbol{r}, \boldsymbol{d}$ and $\Delta \boldsymbol{u}$ are computed by Eqs. (4), (9) and (8) in order. After the convergence of the iteration, $\boldsymbol{u}$ is updated by Eq. (7). There are two major differences between Figs. 2 and 3. The first difference is the position of the matrix factorization. The Newton-Raphson method requires the matrix factorization at every NewtonRaphson iteration, whereas the limited-memory BFGS method computes the matrix factorization once at the beginning of the analysis. The second difference is the presence of vector operations before and after the solution procedure for a linear system of equations. In the limited-memory BFGS method, these vector operations must be computed additionally. At each quasi-Newton iteration step, Eqs. (8) and (9) along with Eq. (11) can be divided into the following procedures:

$$
\begin{aligned}
& \text { 1: } \boldsymbol{r}^{(i)} \leftarrow \boldsymbol{r}\left(\Delta \boldsymbol{u}^{(k, i)}\right) \\
& \text { 2: } \boldsymbol{y}^{(i-1)} \leftarrow \boldsymbol{r}^{(i)}-\boldsymbol{r}^{(i-1)} \\
& \text { 3: } \boldsymbol{\tau}^{(i-1)} \leftarrow \frac{1}{\boldsymbol{d}^{(i-1)} \boldsymbol{y}^{(i-1)}} \\
& \text { 4: } \boldsymbol{p} \leftarrow \boldsymbol{r}^{(i)} \\
& \text { 5: for } j \leftarrow i-1 \text { to } 0 \text { do } \\
& \text { 6: } \quad \gamma^{(j)} \leftarrow \boldsymbol{\tau}^{(j)} \boldsymbol{d}^{(j)^{\mathrm{T}}} \boldsymbol{p}
\end{aligned}
$$




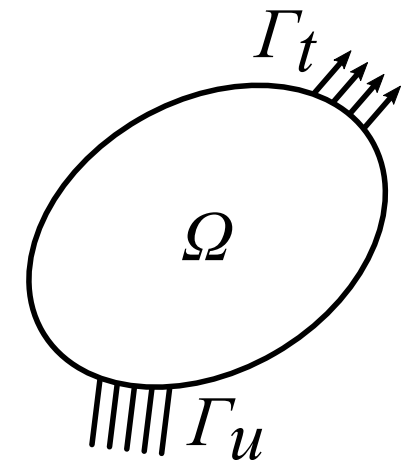

Fig. 1 Domain and its boundary.

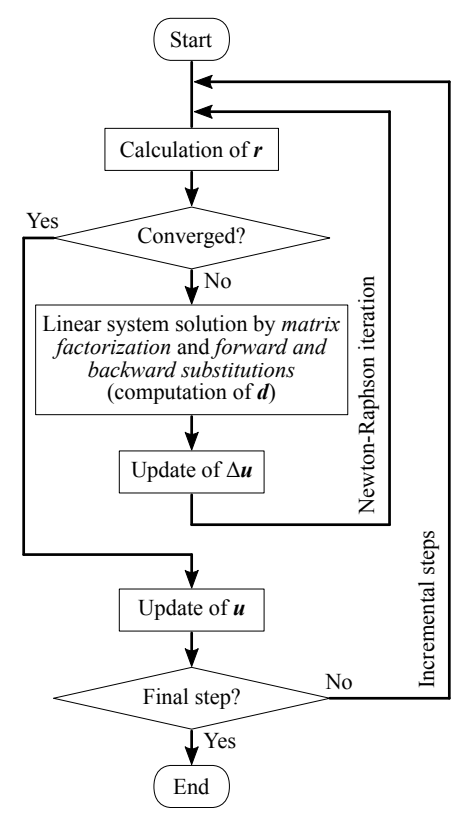

Fig. 2 Flowchart of the incremental method with the Newton-Raphson method.

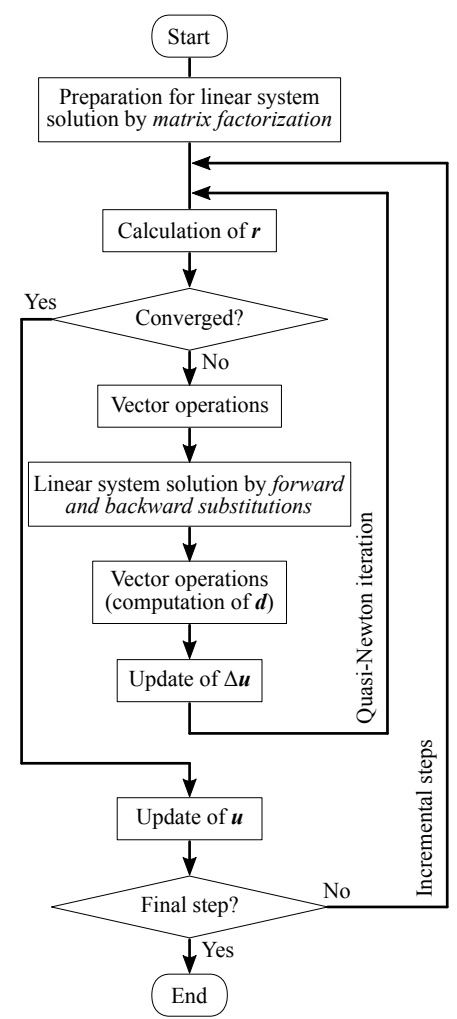

Fig. 3 Flowchart of the incremental method with the limited-memory BFGS method.

7: $\quad \boldsymbol{p} \leftarrow \boldsymbol{p}-\gamma^{(j)} \boldsymbol{y}^{(j)}$

8: end for

9: $\boldsymbol{q} \leftarrow \boldsymbol{K}^{(0)^{-1}} \boldsymbol{p}$

10: for $j \leftarrow 0$ to $i-1$ do

11: $\quad \boldsymbol{q} \leftarrow \boldsymbol{q}+\left(\gamma^{(j)}-\boldsymbol{\tau}^{(j)} \boldsymbol{y}^{(j)}{ }^{\mathrm{T}} \boldsymbol{q}\right) \boldsymbol{d}^{(j)}$

12: end for

13: $\boldsymbol{d}^{(i)} \leftarrow-\boldsymbol{q}$

14: $\Delta \boldsymbol{u}^{(k, i+1)} \leftarrow \Delta \boldsymbol{u}^{(k, i)}+\boldsymbol{d}^{(i)}$

15: $i \leftarrow i+1$

The limited-memory BFGS method stores $\boldsymbol{d}^{(j)}, \boldsymbol{y}^{(j)}, \tau^{(j)}$ and $\gamma^{(j)}$ for $j=0,1, \ldots, i-1$ in the memory space. This is the additional memory consumption of the limited-memory BFGS method as compared to the Newton-Raphson method. However, this additional memory consumption would not be dominant because a sparse direct solver should use much more memory. The space complexity of the four additional variables is $O(n)$ with an assumption that the number of iterations, $i$, is independent from the degrees of freedom, $n$. In contrast, the space complexity of a direct solver is $O\left(n^{2}\right)$ for a dense matrix and $O\left(n^{\frac{5}{3}}\right)$ for a band matrix. Although that of a sparse direct solver depends on its methodology, it is at least greater than $O(n)$. Actual memory consumption will be investigated numerically.

The algorithm of the limited-memory BFGS method includes two typical computational procedures, i.e., a solution procedure for a linear system of equations and vector operations such as vector additions and dot products. In the present framework, the solution procedure for a linear system of equations (9th line) is performed by a distributed-memory parallel sparse direct solver library. Since the coefficient matrix for the linear system of equations, $\boldsymbol{K}^{(0)}$, is the stiffness matrix of an infinitesimal-strain elastic body in the present framework, $\boldsymbol{K}^{(0)}$ remains constant throughout the incremental and quasi-Newton iteration steps. Hence, matrix factorization is performed only once in an analysis as shown in Fig. 3, whereas an increasing frequency of forward and backward substitutions are computed. In contrast, the Newton-Raphson method requires both matrix factorization and forward and backward substitutions at every Newton-Raphson iteration step in every incremental step, as shown in Fig. 2. Then, the vector operations consist of vector additions (11th and 14th lines), vector subtractions (2nd and 7th lines), dot products (3rd, 6th and 11th lines) and scalar-vector multiplications (7th 
and 11th lines). The vector addition and vector subtraction can be computed without communications of message passing interface (MPI). They are computed independently in each subdomain in the present framework. The finite element analysis model is decomposed into subdomains before the analysis. Although both overlapping and non-overlapping types of domain decomposition can be adopted in the present framework, the non-overlapping type was used in the present study. The dot product requires a reduction communication (MP I_Al l reduce) after subdomain-wise dot product computations of subvectors. The use of MPI_Allreduce rather than MPI_Reduce in the dot product computation enables us to omit explicitly calling function of a broadcast communication (MPI_Bcast) in the scalar-vector multiplication. In the scalar-vector multiplication, a scalar that was already broadcasted by MPI_AlIreduce is multiplied by a subvector in each subdomain. The finite element analysis model is decomposed into subdomains before the analysis. Although both overlapping and non-overlapping domain decompositions can be adopted in the present framework, the non-overlapping domain decomposition was used in the present study. The speedup of the quasi-Newton-based present framework over the Newton-Raphson method can roughly be estimated by four typical values. The first value is the computational time ratio between matrix factorization, and forward and backward substitutions, $R$. The second one is the number of incremental steps, $N_{k}$. The third and fourth ones are the number of iterations of the Newton-Raphson method at an incremental step, $N_{i}^{\mathrm{NR}}$, and that of the limited-memory BFGS method, $N_{i}^{\mathrm{BFGS}}$. They are average values for the incremental steps. Because $R, N_{i}^{\mathrm{NR}}$ and $N_{i}^{\mathrm{BFGS}}$ depend on the problem, the three values should be measured in advance. The Newton-Raphson method requires $N_{k} N_{i}^{\mathrm{NR}}$ times of both matrix factorizations and forward and backward substitutions, whereas the limited-memory BFGS method in the present framework needs only one time of matrix factorization and $N_{k} N_{i}^{\mathrm{BFGS}}$ times of forward and backward substitutions. The estimated speedup, $S$, is thus expressed by

$$
S=\frac{(R+1) N_{k} N_{i}^{\mathrm{NR}}}{R+N_{k} N_{i}^{\mathrm{BFGS}} .}
$$

If $R=400, N_{k}=100, N_{i}^{\mathrm{NR}}=3$ and $N_{i}^{\mathrm{BFGS}}=20$, the estimated speedup is 50 . Hence, speedup of tens of times is expected. In this estimation, the contribution of $R$ is especially significant. As the number of DOFs increases, $R$ becomes large in general. Owing to the implementation for parallel computing presented in this subsection, a large number of DOFs became able to be analyzed by the limited-memory BFGS method.

\subsection{Finite-strain elastoplasticity model used in the present framework}

We briefly explain the finite-strain elastoplasticity model that was used in the present study. The Hencky model (de Souza Neto et al., 2008) was used. Although the present framework can be applied to any nonlinear model, we adopted this model as a modern finite-strain elastoplasticity model that is based on the multiplicative decomposition of deformation gradients. In addition, the use of a finite-strain elastoplasticity model would be able to demonstrate the capability of the present framework at least for infinitesimal elastoplasticity models and finite-strain elasticity models. In the Hencky model, the Cauchy stress, $\sigma$, in Eq. (3) is expressed in tensor notation as

$$
\begin{aligned}
\sigma & =\frac{\boldsymbol{\tau}}{\operatorname{det} \boldsymbol{F}}, \\
\boldsymbol{\tau} & =\mathbb{D}:\left(\boldsymbol{\varepsilon}-\boldsymbol{\varepsilon}^{p}\right), \\
\boldsymbol{\varepsilon} & =\frac{1}{2} \ln \left(\boldsymbol{F} \cdot \boldsymbol{F}^{\mathrm{T}}\right), \\
\boldsymbol{F} & =\boldsymbol{I}+\frac{\partial \tilde{\boldsymbol{u}}}{\partial \boldsymbol{x}},
\end{aligned}
$$

where $\boldsymbol{\tau}, \mathbb{D}, \boldsymbol{\varepsilon}, \boldsymbol{F}$ and $\boldsymbol{I}$ denote the Kirchhoff stress, an elasticity tensor, the Hencky strain, a deformation gradient tensor and the identity tensor, respectively. The plastic part of the Hencky strain, $\boldsymbol{\varepsilon}^{p}$, evolves in an isotropic-hardening manner with the von Mises yield criterion. In the implementation, a backward Euler stress integration algorithm with the radial return method was adopted. Moreover, a tangent stiffness matrix that is consistent with the radial return method, $\partial \boldsymbol{r} / \partial \boldsymbol{u}$ in Eq. (10), was implemented for the Newton-Raphson method. Note that the consistent tangent stiffness matrix involving plasticity is not necessary in the limited-memory BFGS method as well as the modified Newton method.

\section{Numerical experiments}

For the investigation of the convergence performance and the speedup of large-deformation elastic-plastic analysis using the present framework, three phases of numerical experiments were arranged. The first phase was a detailed investigation using a finite element analysis model that had 100 thousand DOFs. The mechanism of the speedup is explained 


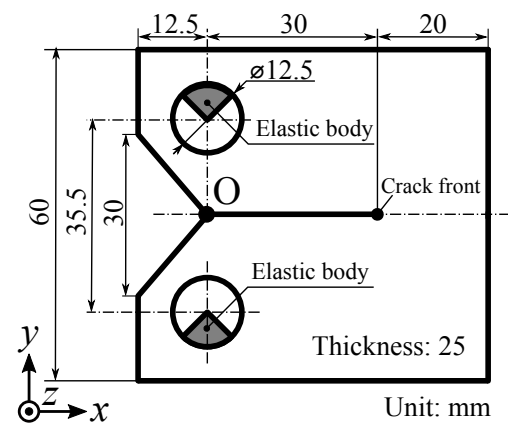

Fig. 4 Dimensions of the crack problem.

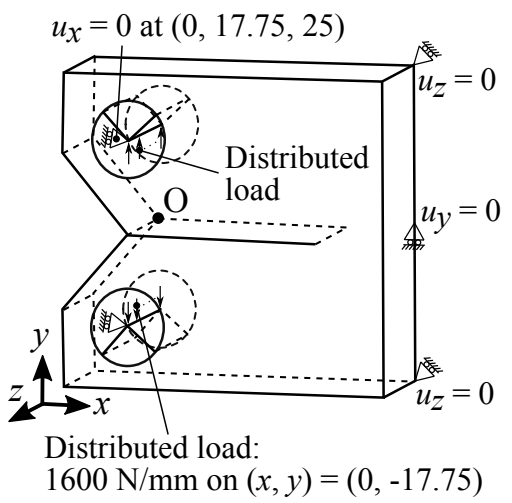

Fig. 5 Boundary conditions for the crack problem.

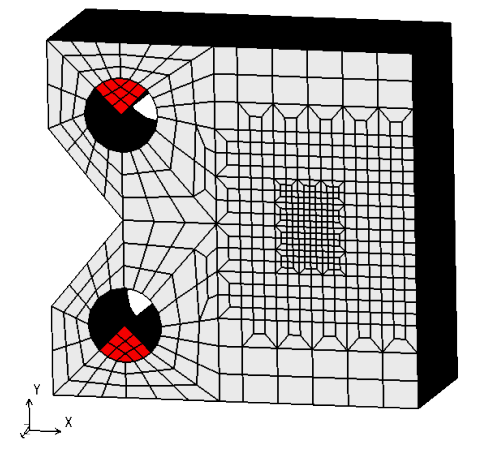

Fig. 6 Finite element analysis model of Fig. 7 Fig. 4.

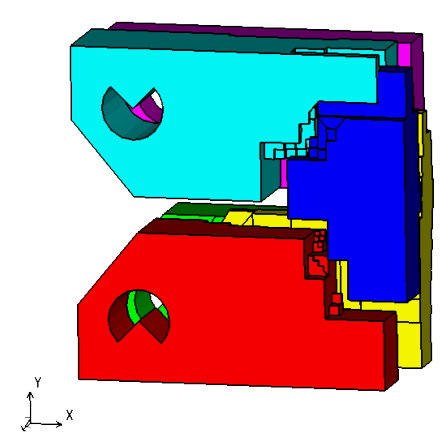

with six subdomains.

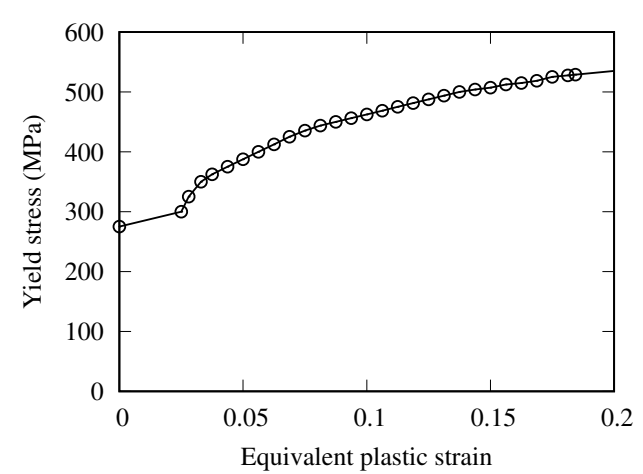

Fig. 8 Yield stress and equivalent plastic strain curve used in the analysis of the crack problem.

based on the numerical results. The second phase was a weak scaling test using multiple finite element analysis models, whose number of DOFs ranged from 100 thousand to 767 thousand. The changes of the convergence performance and the speedup were studied. The final phase was the confirmation of the convergence performance and the speedup. Three examples, each of which involves approximately one million DOFs, were analyzed. The numerical results of the three examples were investigated briefly.

\subsection{Large-deformation elastic-plastic analysis of a crack problem}

We used a crack problem to investigate the computational performance of the present framework. This is because crack problems tend to require a large number of finite elements to reproduce stress and strain concentrations in the vicinity of the crack tip. Moreover, stress and strain concentrations in the vicinity of the crack cause a strongly nonlinear problem that would not be easy to be solved. Among various crack problems, we adopted a compact tension (CT) specimen because its experimental data were openly available from the website of the MDF Subcommittee, Atomic Energy Research Committee, The Japan Welding Engineering Society (2017). This crack problem was solved by three nonlinear solution methods: the Newton-Raphson method, the modified Newton method and the limited-memory BFGS method. The stiffness matrix of the infinitesimal elastic body was used for the initial approximate Jacobian matrix, $\boldsymbol{K}^{(0)}$, in the limited-memory BFGS method. In the modified Newton method, since this stiffness matrix could not achieve convergence, the stiffness matrix of the finite-strain elastic body was used instead. The use of the stiffness matrix of the infinitesimal elastic body requires matrix factorization only once, whereas that of the finite-strain elastic body requires matrix factorization at every incremental step.

The dimensions of the CT specimen are illustrated in Fig. 4. Two load pins were modeled by triangular-shaped elastic bodies. The prescribed boundary conditions are illustrated in Fig. 5. Rigid-body modes are constrained, and distributed loads are incrementally applied at the load pins. The number of incremental steps was set to be 120 . The CT specimen was discretized by finite elements, as depicted in Fig. 6 . The two load pins are colored red. The linear hexahedral finite element was adopted. The volumetric locking behavior was avoided by the B-bar method. The numbers of elements and nodes of this analysis model were 29,800 and 33,354, respectively. The number of DOFs was 100,062. For parallel computing, this analysis model was decomposed into six subdomains by ADVENTURE_Metis 1.1 (ADVENTURE Project, 1997-2021; 


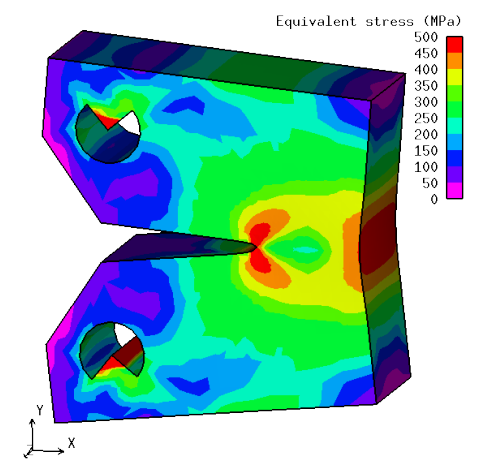

Fig. 9 Distribution of the von Mises equivalent value of the Cauchy stress at the final incremental step in the analysis of of the crack problem.

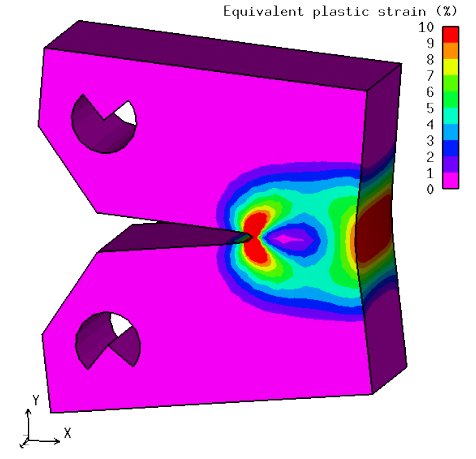

Fig. 10 Distribution of the equivalent plastic strain in the sense strain at the final incremental step in the analysis of of the crack problem.

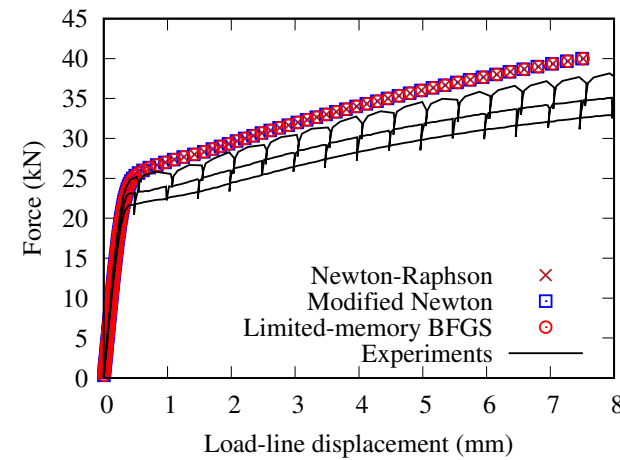

Fig. 11 Relation between the force and the load-line displacement in the analysis of of the crack problem.

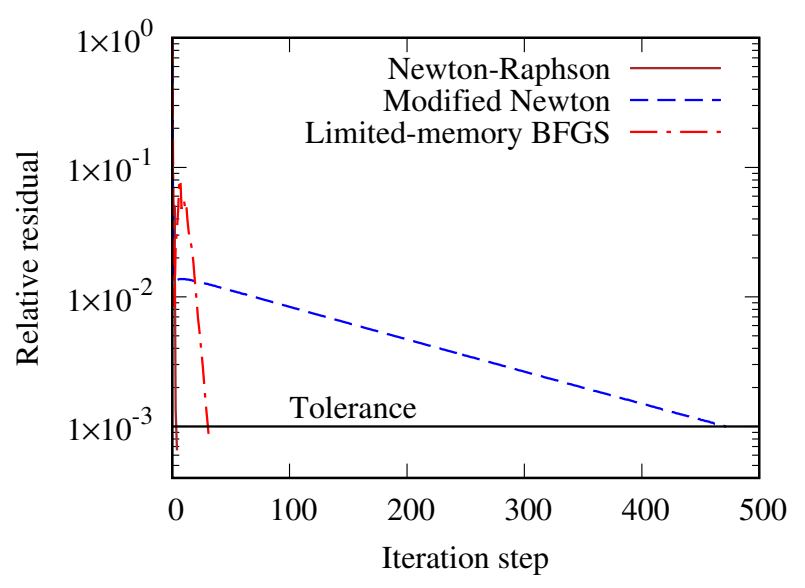

Fig. 12 Convergence histories at the final incremental step in the analysis of of the crack problem.

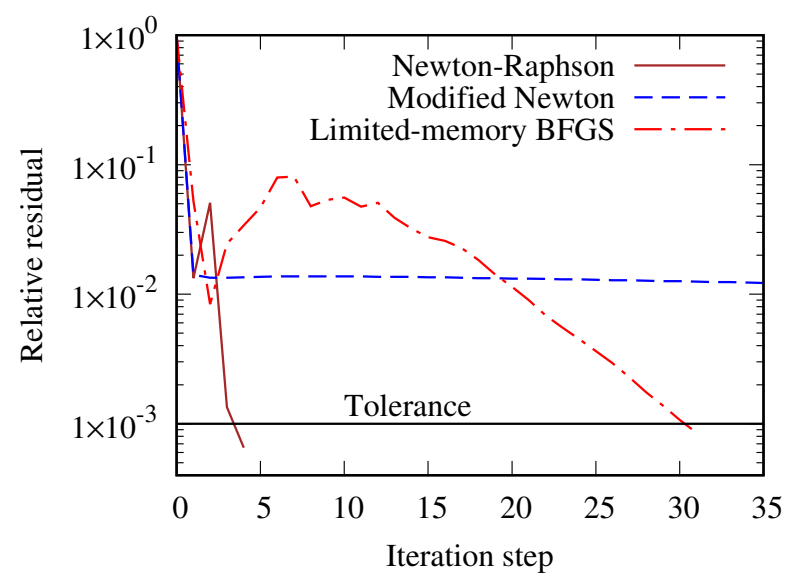

Fig. 13 Enlarged view of Fig. 12.

Yoshimura et al., 2002), as depicted in Fig. 7. ADVENTURE_Metis is a decomposer of a finite element analysis model that is based on the METIS and ParMETIS graph partitioning libraries (Karypis, 2006-2021; Karypis and Kumar, 1998). Each subdomain was assigned to an MPI process. Figure 8 shows the yield stress and equivalent plastic strain curve. The horizontal and vertical axes represent the equivalent plastic strain and the yield stress, respectively. This piece-wise linear curve is based on data published by the MDF Subcommittee, Atomic Energy Research Committee, The Japan Welding Engineering Society (2017). The material was assumed to be SGV410, which is a carbon steel for pressure vessels for intermediate- and moderate-temperature services. Young's modulus and Poisson's ratio of the material are 203 GPa and 0.3, respectively. We used a computer that had an Intel Core i7-8700 CPU and DDR4-2666 memories with 64 GB in total. This CPU had six cores, each of which was dedicated to an MPI process. As a sparse direct solver library, we used MUMPS 5.1.2 (CERFACS et al., 1991-2021; Amestoy et al., 2001, 2019) in conjunction with BLAS 3.8.0, LAPACK 3.8.0, ScaLAPACK 2.0.2, METIS 5.1.0 and ParMETIS 4.0.3.

Figure 9 gives the distribution of the von Mises equivalent value of the Cauchy stress at the final incremental step computed by the limited-memory BFGS method. Moreover, the distribution of equivalent plastic strain is visualized in Fig. 10. Large deformation with crack tip blunting and concentration of stress and strain can be seen. Figure 11 shows the relation between the force and the load-line displacement. The horizontal and vertical axes represent the load-line displacement and the force, respectively. The numerical results of the three nonlinear solution methods are plotted, as well as three experimental data sets. Two of the three experimental data sets include slight unloading and reloading every 0.5 $\mathrm{mm}$ for measuring crack lengths by the unloading elastic compliance method. This figure shows that the numerical results of the three nonlinear solution methods agreed very well. In addition, these numerical results were in good agreement with the experimental results, although they were slightly larger.

The convergence histories of the three nonlinear solution methods at the final incremental step are plotted in Fig. 12, 
Yusa, Miyauchi and Okada, Mechanical Engineering Journal, Vol.8, No.3 (2021)

Table 1 Numbers of processing times of each computational procedure in the analysis of of the crack problem.

\begin{tabular}{|c|c|c|c|}
\hline & Newton-Raphson method & Modified Newton method & Limited-memory BFGS method \\
\hline Coefficient matrix generation & 330 & 120 & 1 \\
\hline Matrix factorization & 330 & 120 & 1 \\
\hline Forward and backward substitutions & 330 & 15,685 & 1,561 \\
\hline Residual vector calculation & 450 & 15,805 & 1,681 \\
\hline Quasi-Newton's vector operations & N/A & N/A & 1,561 \\
\hline
\end{tabular}

Table 2 Total computational time and computational time for each computational procedure in the analysis of the crack problem. Values in parentheses denote percentage of computational time.

\begin{tabular}{rrrrrrr}
\hline & Newton-Raphson method & \multicolumn{4}{c}{ Modified Newton method } & Limited-memory BFGS method \\
\hline Coefficient matrix generation & $535 \mathrm{~s}$ & $(15 \%)$ & $194 \mathrm{~s}$ & $(7 \%)$ & $1 \mathrm{~s}$ & $(1 \%)$ \\
Matrix factorization & $3,061 \mathrm{~s}$ & $(84 \%)$ & $1,116 \mathrm{~s}$ & $(41 \%)$ & $8 \mathrm{~s}$ & $(5 \%)$ \\
Forward and backward substitutions & $18 \mathrm{~s}$ & $(0 \%)$ & $830 \mathrm{~s}$ & $(30 \%)$ & $83 \mathrm{~s}$ & $(52 \%)$ \\
Residual vector calculation & $16 \mathrm{~s}$ & $(0 \%)$ & $594 \mathrm{~s}$ & $(22 \%)$ & $62 \mathrm{~s}$ & $(39 \%)$ \\
Quasi-Newton's vector operations & $\mathrm{N} / \mathrm{A}$ & & $\mathrm{N} / \mathrm{A}$ & & $3 \mathrm{~s}$ & $(2 \%)$ \\
Other procedures & $3 \mathrm{~s}$ & $(0 \%)$ & $10 \mathrm{~s}$ & $(0 \%)$ & $4 \mathrm{~s}$ & $(2 \%)$ \\
\hline Total & $3,633 \mathrm{~s}$ & $(100 \%)$ & $2,744 \mathrm{~s}$ & $(100 \%)$ & $161 \mathrm{~s}$ & $(100 \%)$ \\
\hline
\end{tabular}

an enlarged view of which is shown in Fig. 13. In these figures, the horizontal and vertical axes represent the iteration step and the relative residual, respectively. The relative residual is a 2-norm of $\boldsymbol{r}$ divided by a 2-norm of $\boldsymbol{f}^{\text {ext }}$. The tolerance of the convergence criterion was set to be $10^{-3}$ in the present study. The numbers of iterations of the Newton-Raphson method, the modified Newton method and the limited-memory BFGS method were 4, 472 and 31, respectively. Then, the numbers of processing times of each computational procedure are listed in Table 1. The Newton-Raphson method required 330 times of coefficient matrix generations, matrix factorizations, and forward and backward substitutions. The frequency of residual vector calculations was 450 . The modified Newton method required fewer coefficient matrix generations and matrix factorizations but required far more forward and backward substitutions, and residual vector calculations. The limited-memory BFGS method required coefficient matrix generation and matrix factorization only once. The frequencies of forward and backward substitutions, and residual vector calculations were larger for the limited-memory BFGS method than for the Newton-Raphson method. But they were much smaller than for the modified Newton method. In addition, the limited-memory BFGS method required vector operations such as vector additions and dot products, as shown in Fig. 3.

The total computational time and the computational time for each computational procedure are summarized in Table 2. The Newton-Raphson method had the longest computational time. The modified Newton method and the limitedmemory BFGS method had speedups of 1.32 times and 22.6 times, respectively, as compared with the Newton-Raphson method. In the Newton-Raphson method, 99\% (15\% + 84\%) of computational time was consumed by coefficient matrix generation and matrix factorization. This percentage was reduced to $48 \%(7 \%+41 \%)$ for the modified Newton method and $6 \%(1 \%+5 \%)$ for the limited-memory BFGS method. Instead, $52 \%(30 \%+22 \%)$ and $91 \%(52 \%+39 \%)$ of computational time were used by forward and backward substitutions, and residual vector calculation in the modified Newton method and the limited-memory BFGS method, respectively. The percentage of the vector operations of the quasi-Newton method was only $2 \%$. Therefore, the frequencies of coefficient matrix generation and matrix factorization are key to speedup. Finally, measured memory consumption is presented. The Newton-Raphson method required 1.7 GB memory. This was the same as the modified Newton method. The limited-memory BFGS method used 1.8 GB memory, in which $0.1 \mathrm{~GB}$ was for the vector operations.

\subsection{Weak scaling test of the crack problem}

Using the same problem as in the previous subsection, a weak scaling test was carried out. In this weak scaling test, the number of MPI processes was varied, with each MPI process requiring almost the same number of finite elements. Although a standard weak scaling test investigates the parallel performance, the present study aimed at investigating the changes of the number of iterations, the computational time and the memory consumption. We prepared four finite element analysis models, which are depicted in Fig. 14. The four analysis models are referred to herein as CT specimen \#1, CT specimen \#2, CT specimen \#3 and CT specimen \#4. They were decomposed into subdomains for parallel computing, as shown in Fig. 15. The numbers of MPI processes, subdomains, elements, nodes, DOFs and incremental steps are listed 


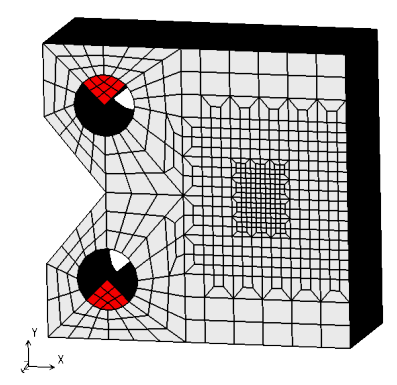

(a) CT specimen \#1

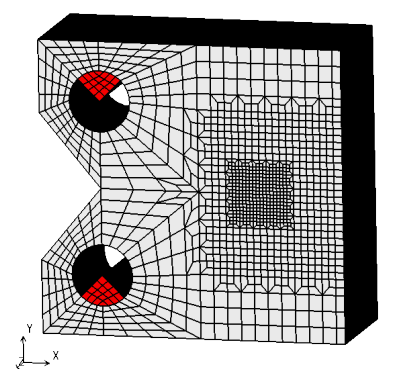

(b) CT specimen \#2

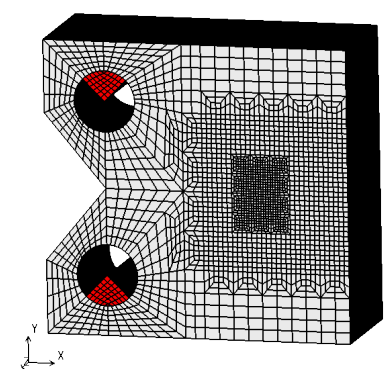

(c) CT specimen \#3

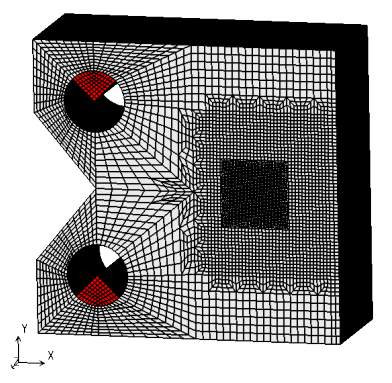

(d) CT specimen \#4

Fig. 14 Finite element analysis models in the weak scaling test of the crack problem.

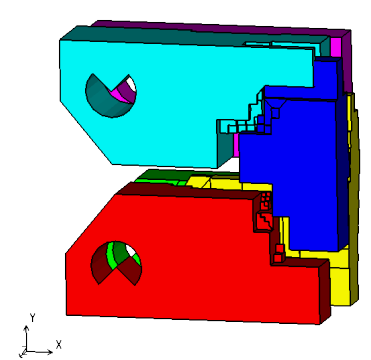

(a) CT specimen \#1 (6 subdomains)

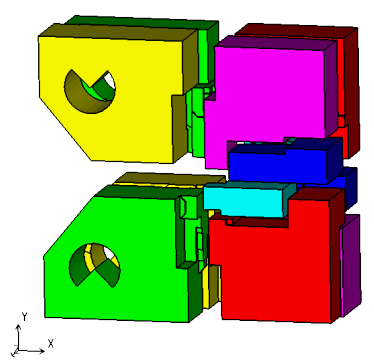

(b) CT specimen \#2 (12 subdomains)

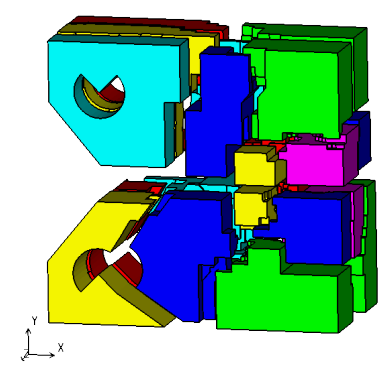

(c) CT specimen \#3 (24 subdomains)

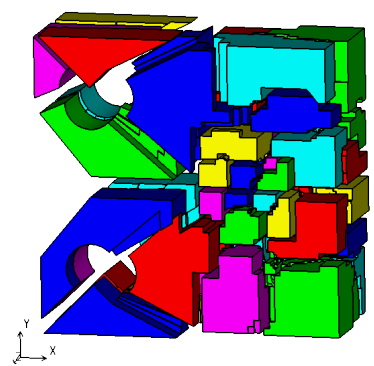

(d) CT specimen \#4 (48 subdomains)

Fig. 15 Domain decomposition of Fig. 14.

Table 3 Numbers of MPI processes, subdomains, elements, nodes, DOFs and incremental steps in the weak scaling test of the crack problem.

\begin{tabular}{rrrrr}
\hline & CT specimen \#1 & CT specimen \#2 & CT specimen \#3 & CT specimen \#4 \\
\hline Number of MPI processes & 6 & 12 & 24 & 48 \\
Number of subdomains & 6 & 12 & 24 & 48 \\
Number of elements & 29,800 & 60,500 & 119,200 & 242,000 \\
Number of nodes & 33,354 & 66,096 & 127,551 & 255,663 \\
Number of DOFs & 100,062 & 198,288 & 382,653 & 766,989 \\
Number of incremental steps & 120 & 120 & 120 & 120 \\
\hline
\end{tabular}

in Table 3. In particular, the number of DOFs ranged from 100 thousand to 767 thousand. For computations, a PC cluster consisting of eight computing nodes connected by 10 Gigabit Ethernet was used. Each computing node had an Intel Core i7-8700 CPU and DDR4-2666 memories with 64 GB. The total number of CPU cores was 48, and the total memory capacity was 512 GB. One, two, four and eight nodes of the PC cluster were used for CT specimen \#1, CT specimen \#2, CT specimen \#3 and CT specimen \#4, respectively.

The numbers of iterations at the final incremental step are listed in Table 4. In addition, the convergence histories of the three nonlinear solution methods at the final incremental step are plotted in Figs. 16, 17 and 18. The horizontal and vertical axes represent the iteration step and the relative residual, respectively. Table 4 and Fig. 16 indicates that the convergence performance of the Newton-Raphson method remained almost the same, even though the number of DOFs changed. In contrast, the number of iterations of the modified Newton method grew gradually with an increasing number of DOFs. Moreover, that of the limited-memory BFGS method increased steeply. In other words, the limited-

Table 4 Numbers of iterations at the final incremental step in the weak scaling test of the crack problem. Values in parentheses denote the maximum number of iterations in the analysis, which is presented if it was larger than the number at the final incremental step.

\begin{tabular}{rrrrr}
\hline & CT specimen \#1 & CT specimen \#2 & CT specimen \#3 & CT specimen \#4 \\
\hline Newton-Raphson method & 4 & $3(5)$ & 5 & 7 \\
Modified Newton method & 472 & 528 & 572 & 604 \\
Limited-memory BFGS method & 31 & 37 & 51 & 92 \\
\hline
\end{tabular}




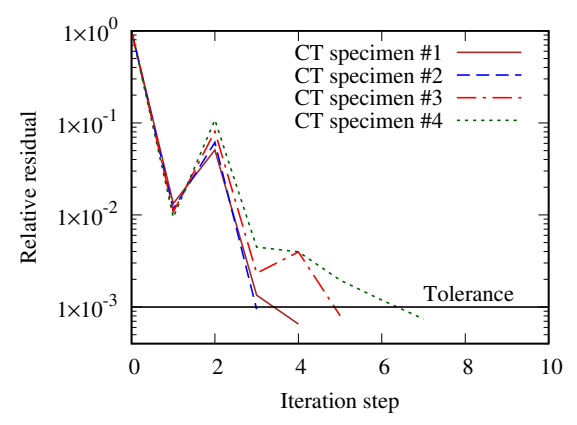

Fig. 16 Convergence histories of the Newton-Raphson method at the final incremental step in the weak scaling test of the crack problem.

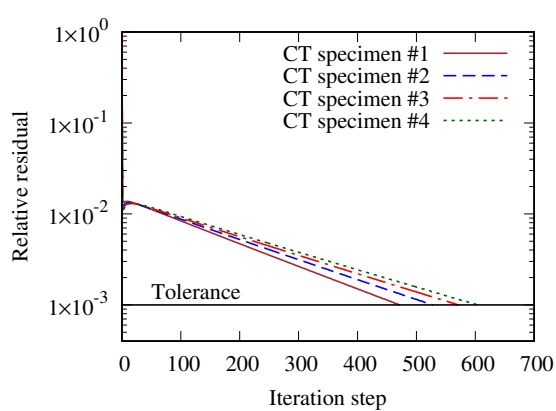

Fig. 17 Convergence histories of the modified Newton method at the final incremental step in the weak scaling test of the crack problem.

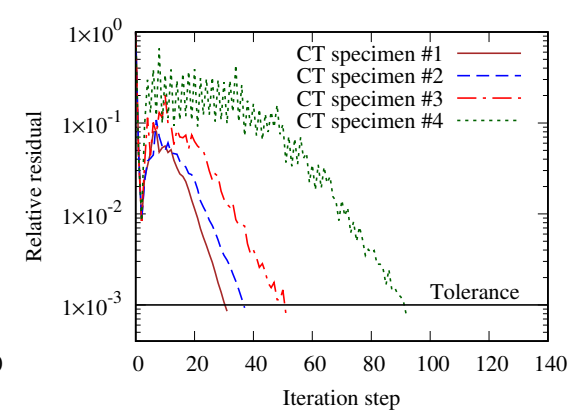

Fig. 18 Convergence histories of the limitedmemory BFGS method at the final incremental step in the weak scaling test of the crack problem.

Table 5 Numbers of processing times of each computational procedure in the weak scaling test of the crack problem.

\begin{tabular}{rrrrr}
\hline Newton-Raphson method & CT specimen \#1 & CT specimen \#2 & CT specimen \#3 & CT specimen \#4 \\
\hline Coefficient matrix generation & 330 & 365 & 379 & 398 \\
Matrix factorization & 330 & 365 & 379 & 398 \\
Forward and backward substitutions & 330 & 365 & 379 & 398 \\
Residual vector calculation & 450 & 485 & 499 & 518 \\
\hline Modified Newton method & CT specimen \#1 & CT specimen \#2 & CT specimen \#3 & CT specimen \#4 \\
\hline Coefficient matrix generation & 120 & 120 & 120 & 120 \\
Matrix factorization & 120 & 120 & 120 & 120 \\
Forward and backward substitutions & 15,685 & 17,152 & 18,333 & 19,499 \\
Residual vector calculation & 15,805 & 17,272 & 18,453 & 19,619 \\
\hline Limited-memory BFGS method & CT specimen \#1 & CT specimen \#2 & CT specimen \#3 & CT specimen \#4 \\
\hline Coefficient matrix generation & 1 & 1 & 1 & 1 \\
Matrix factorization & 1 & 1 & 1 & 1 \\
Forward and backward substitutions & 1,561 & 1,775 & 1,998 & 2,441 \\
Residual vector calculation & 1,681 & 1,895 & 2,118 & 2,561 \\
Quasi-Newton's vector operations & 1,561 & 1,775 & 1,998 & 2,441 \\
\hline
\end{tabular}

memory BFGS method is far from a scalable iterative solver in the sense of the number of iterations. Then, the numbers of processing times of each computational procedure are listed in Table 5. The Newton-Raphson method required several hundred times of coefficient matrix generations, matrix factorizations, forward and backward substitutions, and residual vector calculations for each analysis. The frequency of each procedure did not change very much, although it increased slightly with an increasing number of DOFs. In the modified Newton method, the frequencies of coefficient matrix generations and matrix factorizations stayed to be 120 , which is equal to the number of incremental steps. In contrast, the frequencies of forward and backward substitutions, and residual vector calculations increased gradually from 16 thousand to 20 thousand. In the limited-memory BFGS method, coefficient matrix generation and matrix factorization were performed once for each. The frequencies of forward and backward substitutions, and residual vector calculations increased gradually from 1.6 thousand to 2.6 thousand, which were nearly one tenth of those of the modified Newton method.

The total computational time and the computational time for each computational procedure are summarized in Table 6. The total computational time in Table 6 is plotted in Fig. 19, an enlarged view of which is shown in Fig. 20. In these figures, the horizontal and vertical axes represent the number of MPI processes and the computational time, respectively. Although the modified Newton method was 1.32 times faster than the Newton-Raphson method in the analysis of CT specimen \#1, it grew into 2.12 times for CT specimen \#4. Similarly, the speedups of the limited-memory BFGS method were 22.6 times for CT specimen \#1 and 46.7 times for CT specimen \#4. The reason why speedup changed significantly can be found in the computational time for each computational procedure. The Newton-Raphson method consumed approximately $99 \%$ of computational time for coefficient matrix generation and matrix factorization for every analysis. In particular, the percentage of matrix factorization expanded as the number of DOFs increased. This is because matrix factorization requires time complexity that is a higher-degree polynomial of the number of DOFs, than coefficient matrix generation. In the modified Newton method with CT specimen $\# 1,48 \%(7 \%+41 \%)$ of computational time was 
Table 6 Total computational time and computational time for each computational procedure in the weak scaling test of the crack problem. Values in parentheses denote percentage of computational time.

\begin{tabular}{rrrrrrrrr}
\hline Newton-Raphson method & CT specimen \#1 & CT specimen \#2 & CT specimen \#3 & CT specimen \#4 \\
\hline Coefficient matrix generation & $535 \mathrm{~s}$ & $(15 \%)$ & $611 \mathrm{~s}$ & $(8 \%)$ & $620 \mathrm{~s}$ & $(4 \%)$ & $664 \mathrm{~s}$ & $(2 \%)$ \\
Matrix factorization & $3,061 \mathrm{~s}$ & $(84 \%)$ & $6,612 \mathrm{~s}$ & $(91 \%)$ & $15,801 \mathrm{~s}$ & $(96 \%)$ & $39,189 \mathrm{~s}$ & $(98 \%)$ \\
Forward and backward substitutions & $18 \mathrm{~s}$ & $(0 \%)$ & $32 \mathrm{~s}$ & $(0 \%)$ & $55 \mathrm{~s}$ & $(0 \%)$ & $104 \mathrm{~s}$ & $(0 \%)$ \\
Residual vector calculation & $16 \mathrm{~s}$ & $(0 \%)$ & $18 \mathrm{~s}$ & $(0 \%)$ & $19 \mathrm{~s}$ & $(0 \%)$ & $21 \mathrm{~s}$ & $(0 \%)$ \\
Other procedures & $3 \mathrm{~s}$ & $(0 \%)$ & $4 \mathrm{~s}$ & $(0 \%)$ & $5 \mathrm{~s}$ & $(0 \%)$ & $10 \mathrm{~s}$ & $(0 \%)$ \\
\hline Total & $3,633 \mathrm{~s}$ & $(100 \%)$ & $7,277 \mathrm{~s}$ & $(100 \%)$ & $16,500 \mathrm{~s}(100 \%)$ & $39,988 \mathrm{~s}(100 \%)$ \\
\hline Modified Newton method & CT specimen \#1 & CT specimen \#2 & CT specimen \#3 & CT specimen \#4 \\
\hline Coefficient matrix generation & $194 \mathrm{~s}$ & $(7 \%)$ & $201 \mathrm{~s}$ & $(4 \%)$ & $196 \mathrm{~s}$ & $(2 \%)$ & $200 \mathrm{~s}$ & $(1 \%)$ \\
Matrix factorization & $1,116 \mathrm{~s}$ & $(41 \%)$ & $2,168 \mathrm{~s}$ & $(48 \%)$ & $4,708 \mathrm{~s}$ & $(57 \%)$ & $12,833 \mathrm{~s}$ & $(68 \%)$ \\
Forward and backward substitutions & $830 \mathrm{~s}$ & $(30 \%)$ & $1,470 \mathrm{~s}$ & $(32 \%)$ & $2,619 \mathrm{~s}$ & $(32 \%)$ & $4,952 \mathrm{~s}$ & $(26 \%)$ \\
Residual vector calculation & $594 \mathrm{~s}$ & $(22 \%)$ & $677 \mathrm{~s}$ & $(15 \%)$ & $739 \mathrm{~s}$ & $(9 \%)$ & $807 \mathrm{~s}$ & $(4 \%)$ \\
Other procedures & $10 \mathrm{~s}$ & $(0 \%)$ & $13 \mathrm{~s}$ & $(0 \%)$ & $21 \mathrm{~s}$ & $(0 \%)$ & $33 \mathrm{~s}$ & $(0 \%)$ \\
\hline Total & $2,744 \mathrm{~s}$ & $(100 \%)$ & $4,529 \mathrm{~s}$ & $(100 \%)$ & $8,283 \mathrm{~s}(100 \%)$ & $18,825 \mathrm{~s}(100 \%)$ \\
\hline Limited-memory BFGS method & $\mathrm{CT} \mathrm{specimen} \mathrm{\# 1}$ & CT specimen \#2 & CT specimen \#3 & CT specimen \#4 \\
\hline Coefficient matrix generation & $1 \mathrm{~s}$ & $(1 \%)$ & $2 \mathrm{~s}$ & $(1 \%)$ & $1 \mathrm{~s}$ & $(0 \%)$ & $2 \mathrm{~s}$ & $(0 \%)$ \\
Matrix factorization & $8 \mathrm{~s}$ & $(5 \%)$ & $17 \mathrm{~s}$ & $(7 \%)$ & $38 \mathrm{~s}$ & $(9 \%)$ & $107 \mathrm{~s}$ & $(13 \%)$ \\
Forward and backward substitutions & $83 \mathrm{~s}$ & $(52 \%)$ & $151 \mathrm{~s}$ & $(60 \%)$ & $290 \mathrm{~s}$ & $(67 \%)$ & $610 \mathrm{~s}$ & $(71 \%)$ \\
Residual vector calculation & $62 \mathrm{~s}$ & $(39 \%)$ & $73 \mathrm{~s}$ & $(29 \%)$ & $84 \mathrm{~s}$ & $(20 \%)$ & $105 \mathrm{~s}$ & $(12 \%)$ \\
Quasi-Newton's vector operations & $3 \mathrm{~s}$ & $(2 \%)$ & $5 \mathrm{~s}$ & $(2 \%)$ & $10 \mathrm{~s}$ & $(2 \%)$ & $22 \mathrm{~s}$ & $(3 \%)$ \\
Other procedures & $4 \mathrm{~s}$ & $(2 \%)$ & $5 \mathrm{~s}$ & $(2 \%)$ & $7 \mathrm{~s}$ & $(2 \%)$ & $10 \mathrm{~s}$ & $(1 \%)$ \\
\hline Total & $161 \mathrm{~s}$ & $(100 \%)$ & $253 \mathrm{~s}$ & $(100 \%)$ & $430 \mathrm{~s}(100 \%)$ & $856 \mathrm{~s}(100 \%)$ \\
\hline
\end{tabular}

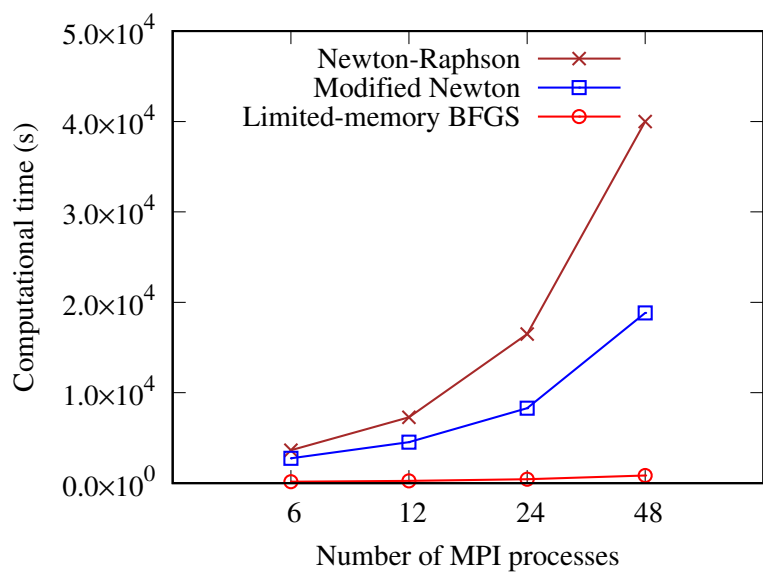

Fig. 19 Graph of total computational time in Table 6.

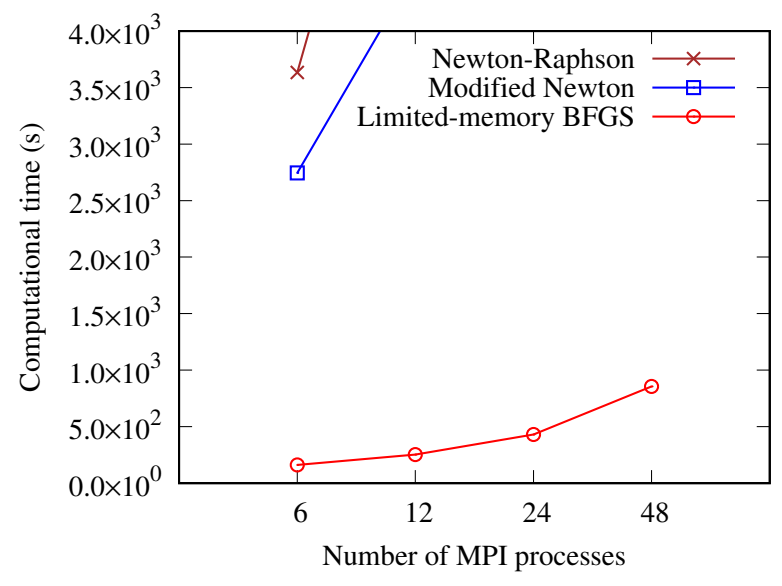

Fig. 20 Enlarged view of Fig. 19.

used for coefficient matrix generation and matrix factorization, whereas $52 \%(30 \%+22 \%)$ was dedicated to forward and backward substitutions, and residual vector calculation. The former one grew to $69 \%(1 \%+68 \%)$ and the latter one was reduced to $30 \%(26 \%+4 \%)$, respectively, for CT specimen \#4. This is also due to the high-degree polynomial of matrix factorization. Similarly, in the limited-memory BFGS method with CT specimen \#1, the former percentage was $6 \%(1 \%+5 \%)$, whereas the latter percentage was $91 \%(52 \%+39 \%)$. They became $13 \%(0 \%+13 \%)$ and $83 \%(71 \%+$ $12 \%$ ), respectively, for CT specimen \#4. In addition, quasi-Newton's vector operations required $2 \%-3 \%$ of computational time for every analysis. In Table 6, the coefficient matrix generation, the residual vector calculation and quasi-Newton's vector operations should have scalability. The computational time of the coefficient matrix generation stayed almost the same. However, the residual vector calculation required gradually increasing computational time as the number of DOFs increased. This is because the number of iterations gradually grew as discussed in the previous paragraph. Furthermore, the computational time of quasi-Newton's vector operations increased steeply. This is probably due to frequent reduction communications in the algorithm as explained in $\S 2.2$. Finally, Table 7 gives measured memory consumption. Memory consumption grew steeply as the number of DOFs increased. This is due to the high-degree polynomial of memory consumption of a direct linear solution method. In addition, the additional memory consumption of the limited-memory BFGS method was at most approximately $5 \%$. 
Table 7 Measured memory consumption in the weak scaling test of the crack problem.

\begin{tabular}{rrrrr}
\hline & CT specimen \#1 & CT specimen \#2 & CT specimen \#3 & CT specimen \#4 \\
\hline Newton-Raphson method & $1.7 \mathrm{~GB}$ & $5.8 \mathrm{~GB}$ & $12.5 \mathrm{~GB}$ & $35.8 \mathrm{~GB}$ \\
Modified Newton method & $1.7 \mathrm{~GB}$ & $5.8 \mathrm{~GB}$ & $12.7 \mathrm{~GB}$ & $35.3 \mathrm{~GB}$ \\
Limited-memory BFGS method & $1.8 \mathrm{~GB}$ & $5.9 \mathrm{~GB}$ & $12.9 \mathrm{~GB}$ & $36.9 \mathrm{~GB}$ \\
\hline
\end{tabular}

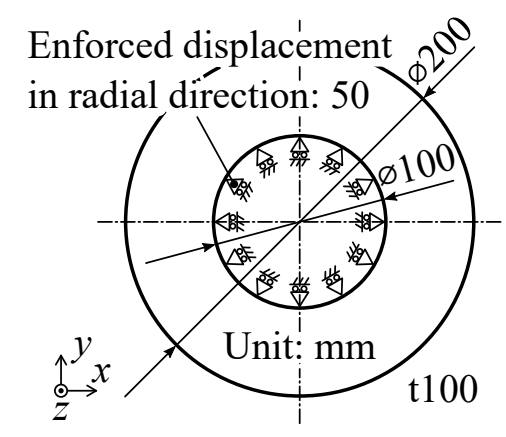

(a) Cylinder

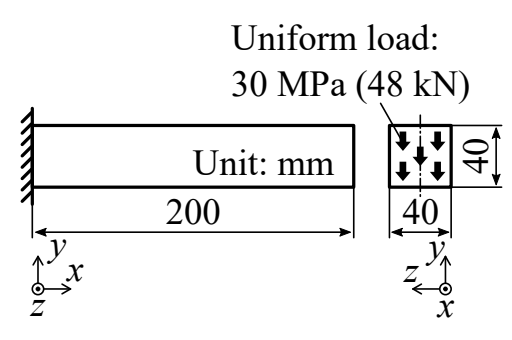

(b) Cantilever beam

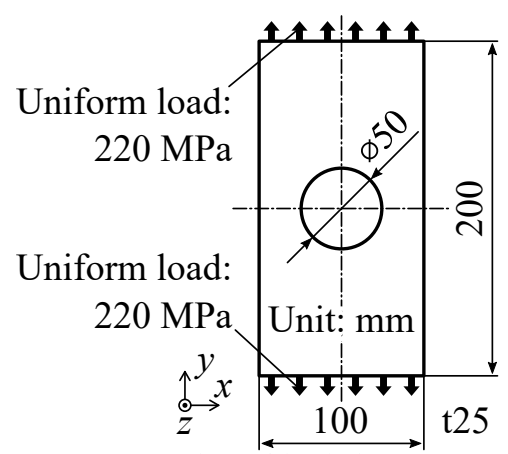

(c) Plate with a hole

Fig. 21 Dimensions and boundary conditions of the three examples.

\subsection{Examples of large-deformation elastic-plastic analyses involving approximately one million DOFs}

To confirm the convergence performance and the speedup, three examples were analyzed. Every example had approximately one million DOFs, which was slightly larger than CT specimen \#4. The three examples were (a) a cylinder, (b) a cantilever beam and (c) a plate with a hole. Their dimensions and boundary conditions are illustrated in Fig. 21. (a) The cylinder under internal pressure is the most basic problem in plasticity. The internal pressure was modeled by enforced displacements in the $x$ and $y$ directions. In addition, a single DOF in the $z$ direction was constrained to prevent rigid-body motion. (b) The cantilever beam is a bending deformation problem. It was subjected to uniform shear load on the surface of the free end. (c) The plate with a hole under uniform tension is a typical stress concentration problem. Uniform loads were prescribed on the top and bottom surfaces of the plate. Moreover, six DOFs of rigid-body modes were constrained.

Finite element analysis models of the three examples are depicted in Fig. 22. Since overall views are filled with mesh lines, enlarged views are presented. The three finite element analysis models were decomposed for parallel computing into 48 subdomains, as shown in Fig. 23. Each of the 48 subdomains was assigned to an MPI process. The number of MPI processes, subdomains, elements, nodes, DOFs and incremental steps are summarized in Table 8 . The material was assumed to be the same as that in the previous subsection, namely, SGV410. This is because it is a typical carbon steel. The PC cluster with eight computing nodes was again used.

Figure 24 gives the distributions of equivalent plastic strain at the final incremental step computed by the limitedmemory BFGS method. Every analysis result was largely deformed under the plastic state. The maximum values of equivalent plastic strain of the three examples were $43.6 \%, 86.6 \%$ and $29.5 \%$, respectively. All of them exceeded $20 \%$, which is the maximum value of the horizontal axis of Fig. 8.

The numbers of iterations of the three nonlinear solution methods are listed in Table 9. Moreover, the numbers of processing times of each computational procedure are summarized in Table 10. Similarly to the previous subsection, the modified Newton method required less frequencies of coefficient matrix generation and matrix factorization, compared to the Newton-Raphson method. In the limited-memory BFGS method, they were performed only once for each. Instead, the frequencies of forward and backward substitutions, and residual vector calculation were much larger in both the two methods, compared to the Newton-Raphson method. This is due to increasing numbers of iterations.

The total computational time and the computational time for each computational procedure are summarized in Table 11. The percentages of each computational procedure were similar to that of CT specimen \#4 in the previous subsection. The speedups of the modified Newton method over the Newton-Raphson method for the three examples were 2.89 times, 2.57 times and 2.29 times, respectively. Those of the limited-memory BFGS method were 39.0 times, 35.9 times and 37.4 times, respectively. The reason why these speedups are slightly lower than that of CT specimen \#4 is due to smaller numbers of incremental steps. When the number of incremental steps is set to be large, the percentage of matrix factorization in computational time shrinks. Finally, Table 12 gives measured memory consumption. Additional memory consumption of the limited-memory BFGS method was negligible. 


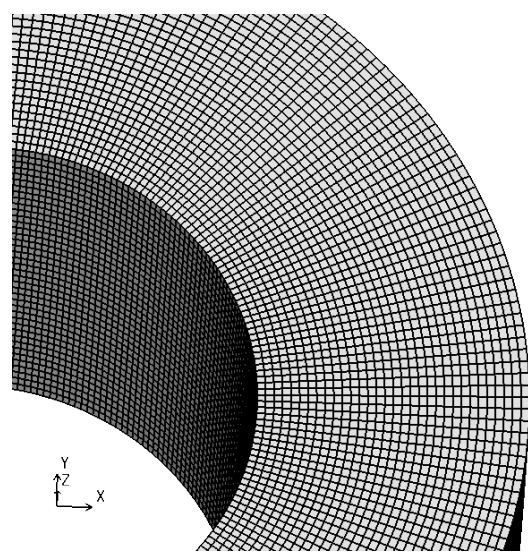

(a) Cylinder

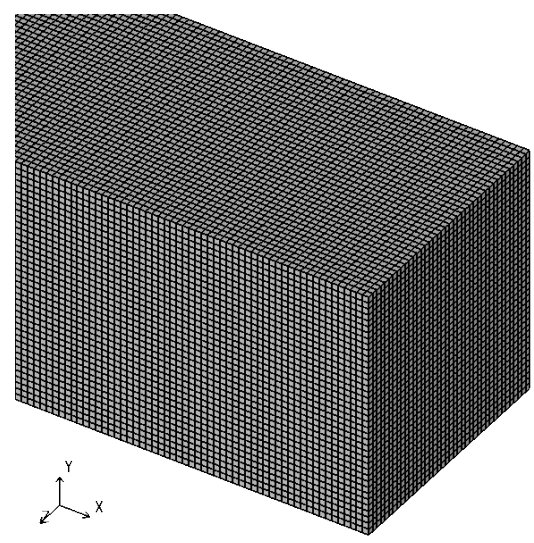

(b) Cantilever beam

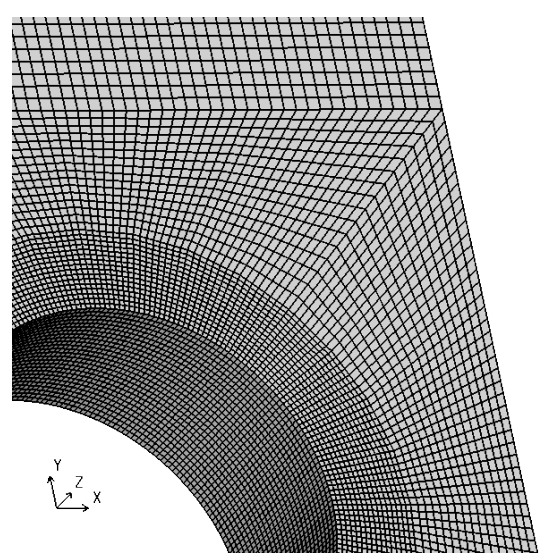

(c) Plate with a hole

Fig. 22 Finite element analysis models of Fig. 21.

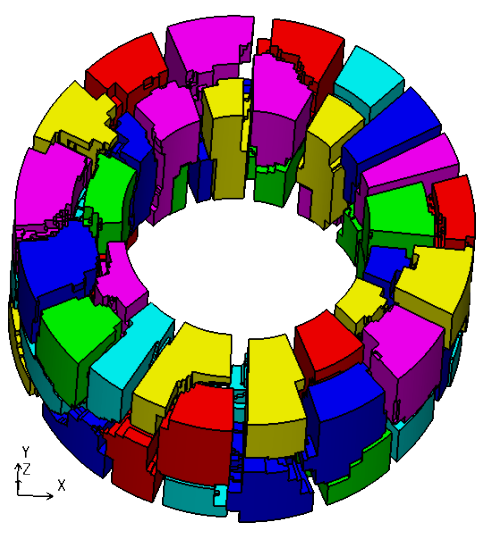

(a) Cylinder

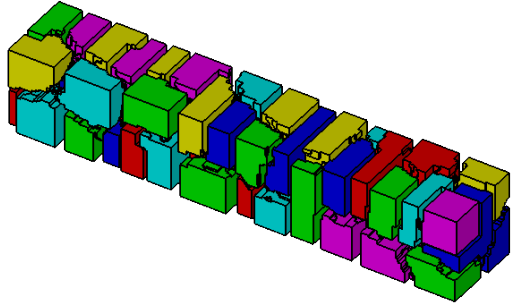

$\stackrel{L}{x} x_{x}$

(b) Cantilever beam

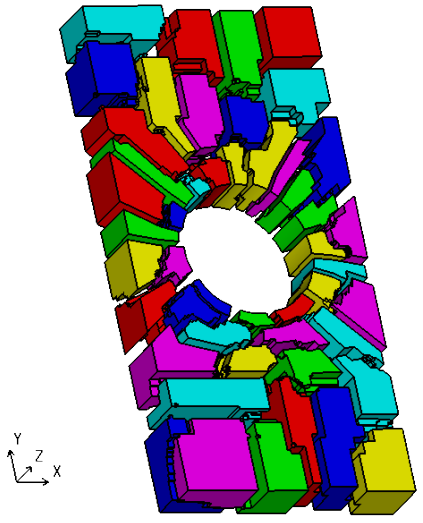

(c) Plate with a hole

Fig. 23 Domain decomposition of Fig. 22 with 48 subdomains for each analysis model.

\section{Conclusion}

We investigated the computational performance of the present framework of a quasi-Newton-based parallel nonlinear finite element method consisting of the limited-memory BFGS method, implementation for distributed-memory parallel computer environments and a modern finite-strain elastoplasticity model. The quasi-Newton-based present framework achieved speedup of more than 30 times for approximately one million DOFs, compared to the Newton-Raphson method. For example, the Newton-Raphson method required 11.1 hours for the analysis with 767 thousand DOFs. This computational time was reduced by the quasi-Newton-based present framework to 14.3 minutes. The measured speedups roughly agreed with the estimated speedup. Furthermore, speedup grew for an increasing number of DOFs: 22.6 times for 100 thousand DOFs and 46.7 times for 767 thousand DOFs. Such quasi-Newton-based analyses over 100 thousand DOFs were enabled by the implementation of the limited-memory BFGS method for distributed-memory parallel computer environments and the use of a parallel sparse direct solver library. In addition, the speedups were measured in practical large-deformation elastic-plastic analyses using a modern finite-strain elastoplasticity model with a realistic multilinear

Table 8 Numbers of MPI processes, subdomains, elements, nodes, DOFs and incremental steps in the three examples.

\begin{tabular}{rrrr}
\hline & Cylinder & Cantilever beam & Plate with a hole \\
\hline Number of MPI processes & 48 & 48 & 48 \\
Number of subdomains & 48 & 48 & 48 \\
Number of elements & 327,680 & 320,000 & 307,200 \\
Number of nodes & 346,368 & 337,881 & 327,808 \\
Number of DOFs & $1,039,104$ & $1,013,643$ & 983,424 \\
Number of incremental steps & 30 & 50 & 50 \\
\hline
\end{tabular}




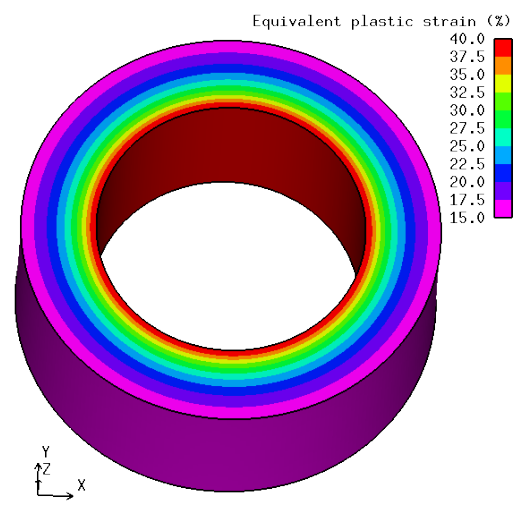

(a) Cylinder

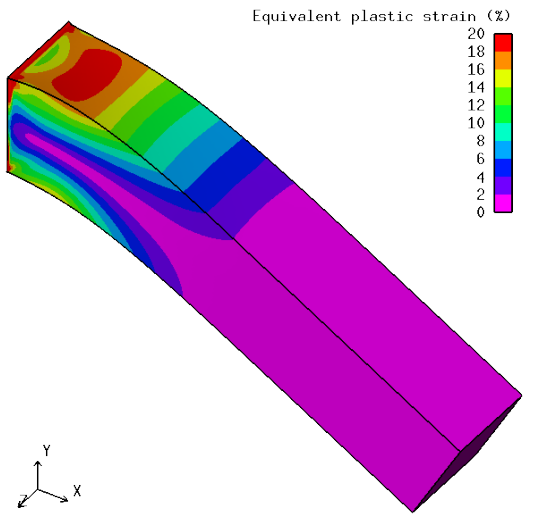

(b) Cantilever beam

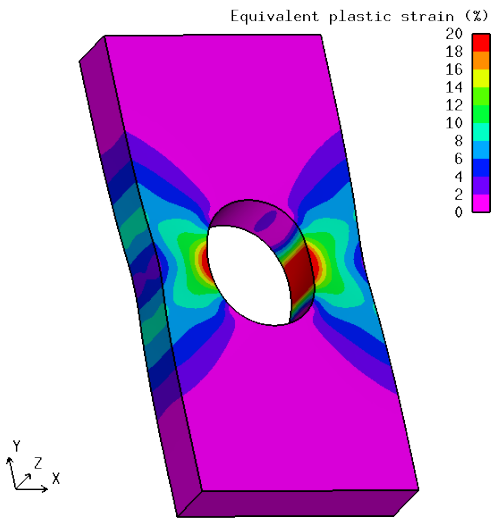

(c) Plate with a hole

Fig. 24 Distribution of the equivalent plastic strain at the final incremental step in the three examples.

Table 9 Numbers of iterations at the final incremental step in the three examples. Values in parentheses denote the maximum number of iterations in the analysis, which is presented if it was larger than the number at the final incremental step.

\begin{tabular}{rrrr}
\hline & Cylinder & Cantilever beam & Plate with a hole \\
\hline Newton-Raphson method & 3 & 5 & 22 \\
Modified Newton method & $15(55)$ & $486(578)$ & 1,063 \\
Limited-memory BFGS method & $16(19)$ & 102 & 70 \\
\hline
\end{tabular}

stress-strain curve. The numerical results in the present paper demonstrated that a nonlinear finite element method that is based on a quasi-Newton method, such as the limited-memory BFGS method, is a good alternative to the NewtonRaphson method. It has the potential to achieve tens of times speedup or more in various nonlinear finite element analyses over 100 thousand DOFs.

The number of iterations of the limited-memory BFGS method was not scalable in $\S 3.2$. For scalability and further speedup, we are trying to apply coarse grid correction to the limited-memory BFGS method. The authors previously proposed a technique to apply coarse grid correction to quasi-Newton methods in the sense of the domain decomposition method (Yusa et al., 2018). This technique will be modified to be combined with the present framework.

\section{Acknowledgement}

The present study was supported by REDAS (Fundamental Research Developing Association for Shipbuilding and Offshore) and JSPS KAKENHI Grant Number JP19K14871.

Table 10 Numbers of processing times of each computational procedure in the three examples.

\begin{tabular}{rrrr}
\hline Newton-Raphson method & Cylinder & Cantilever beam & Plate with a hole \\
\hline Coefficient matrix generation & 90 & 192 & 179 \\
Matrix factorization & 90 & 192 & 179 \\
Forward and backward substitutions & 90 & 192 & 179 \\
Residual vector calculation & 120 & 242 & 229 \\
\hline Modified Newton method & Cylinder & Cantilever beam & Plate with a hole \\
\hline Coefficient matrix generation & 30 & 50 & 50 \\
Matrix factorization & 30 & 50 & 50 \\
Forward and backward substitutions & 737 & 10,236 & 9,401 \\
Residual vector calculation & 767 & 10,286 & 9,451 \\
\hline Limited-memory BFGS method & Cylinder & Cantilever beam & Plate with a hole \\
\hline Coefficient matrix generation & 1 & 1 & 1 \\
Matrix factorization & 1 & 1 & 1 \\
Forward and backward substitutions & 452 & 1,645 & 1,096 \\
Residual vector calculation & 482 & 1,695 & 1,146 \\
Quasi-Newton's vector operations & 452 & 1,645 & 1,096 \\
\hline
\end{tabular}


Table 11 Total computational time and computational time for each computational procedure in the three examples. Values in parentheses denote percentage of computational time.

\begin{tabular}{rrrrrrr}
\hline Newton-Raphson method & \multicolumn{4}{c}{ Cylinder } & \multicolumn{4}{c}{ Cantilever beam } & \multicolumn{2}{r}{ Plate with a hole } \\
\hline Coefficient matrix generation & $201 \mathrm{~s}$ & $(2 \%)$ & $420 \mathrm{~s}$ & $(1 \%)$ & $379 \mathrm{~s}$ & $(2 \%)$ \\
Matrix factorization & $10,805 \mathrm{~s}$ & $(98 \%)$ & $29,427 \mathrm{~s}$ & $(98 \%)$ & $16,082 \mathrm{~s}$ & $(97 \%)$ \\
Forward and backward substitutions & $25 \mathrm{~s}$ & $(0 \%)$ & $68 \mathrm{~s}$ & $(0 \%)$ & $46 \mathrm{~s}$ & $(0 \%)$ \\
Residual vector calculation & $7 \mathrm{~s}$ & $(0 \%)$ & $13 \mathrm{~s}$ & $(0 \%)$ & $12 \mathrm{~s}$ & $(0 \%)$ \\
Other procedures & $8 \mathrm{~s}$ & $(0 \%)$ & $10 \mathrm{~s}$ & $(0 \%)$ & $17 \mathrm{~s}$ & $(0 \%)$ \\
\hline Total & $11,046 \mathrm{~s}(100 \%)$ & $29,938 \mathrm{~s}(100 \%)$ & $16,527 \mathrm{~s}(100 \%)$ \\
\hline Modified Newton method & \multicolumn{1}{c}{ Cylinder } & Cantilever beam & Plate with a hole \\
\hline Coefficient matrix generation & $67 \mathrm{~s}$ & $(2 \%)$ & $109 \mathrm{~s}$ & $(1 \%)$ & $106 \mathrm{~s}$ & $(1 \%)$ \\
Matrix factorization & $3,498 \mathrm{~s}$ & $(91 \%)$ & $7,569 \mathrm{~s}$ & $(65 \%)$ & $4,248 \mathrm{~s}$ & $(59 \%)$ \\
Forward and backward substitutions & $204 \mathrm{~s}$ & $(5 \%)$ & $3,400 \mathrm{~s}$ & $(29 \%)$ & $2,323 \mathrm{~s}$ & $(32 \%)$ \\
Residual vector calculation & $45 \mathrm{~s}$ & $(1 \%)$ & $568 \mathrm{~s}$ & $(5 \%)$ & $513 \mathrm{~s}$ & $(7 \%)$ \\
Other procedures & $9 \mathrm{~s}$ & $(0 \%)$ & $21 \mathrm{~s}$ & $(0 \%)$ & $20 \mathrm{~s}$ & $(0 \%)$ \\
\hline Total & $3,823 \mathrm{~s}(100 \%)$ & $11,667 \mathrm{~s}(100 \%)$ & $7,210 \mathrm{~s}(100 \%)$ \\
\hline Limited-memory BFGS method & \multicolumn{2}{c}{ Cylinder } & Cantilever beam & Plate with a hole \\
\hline Coefficient matrix generation & $2 \mathrm{~s}$ & $(1 \%)$ & $2 \mathrm{~s}$ & $(0 \%)$ & $2 \mathrm{~s}$ & $(0 \%)$ \\
Matrix factorization & $119 \mathrm{~s}$ & $(42 \%)$ & $148 \mathrm{~s}$ & $(18 \%)$ & $88 \mathrm{~s}$ & $(20 \%)$ \\
Forward and backward substitutions & $123 \mathrm{~s}$ & $(43 \%)$ & $553 \mathrm{~s}$ & $(66 \%)$ & $269 \mathrm{~s}$ & $(61 \%)$ \\
Residual vector calculation & $28 \mathrm{~s}$ & $(10 \%)$ & $94 \mathrm{~s}$ & $(11 \%)$ & $61 \mathrm{~s}$ & $(14 \%)$ \\
Quasi-Newton's vector operations & $2 \mathrm{~s}$ & $(1 \%)$ & $25 \mathrm{~s}$ & $(3 \%)$ & $12 \mathrm{~s}$ & $(3 \%)$ \\
Other procedures & $9 \mathrm{~s}$ & $(3 \%)$ & $11 \mathrm{~s}$ & $(1 \%)$ & $10 \mathrm{~s}$ & $(2 \%)$ \\
\hline Total & $283 \mathrm{~s}(100 \%)$ & $833 \mathrm{~s}(100 \%)$ & $442 \mathrm{~s}(100 \%)$ \\
\hline
\end{tabular}

Table 12 Measured memory consumption in the three examples.

\begin{tabular}{rrrr}
\hline & Cylinder & Cantilever beam & Plate with a hole \\
\hline Newton-Raphson method & $39.4 \mathrm{~GB}$ & $42.4 \mathrm{~GB}$ & $35.3 \mathrm{~GB}$ \\
Modified Newton method & $39.5 \mathrm{~GB}$ & $42.4 \mathrm{~GB}$ & $35.3 \mathrm{~GB}$ \\
Limited-memory BFGS method & $39.1 \mathrm{~GB}$ & $43.7 \mathrm{~GB}$ & $36.5 \mathrm{~GB}$ \\
\hline
\end{tabular}

\section{References}

ADVENTURE Project, Development of computational mechanics system for large scale analysis and design (online) (1997-2021), <https://adventure.sys.t.u-tokyo.ac.jp/>, (accessed on 5 January 2021).

Amestoy, P., Duff, I., L'Excellent, J. and Koster, J., A fully asynchronous multifrontal solver using distributed dynamic scheduling, SIAM Journal on Matrix Analysis and Applications, Vol. 23, No. 1 (2001), pp. 15-41.

Amestoy, P. R., Buttari, A., L'Excellent, J.-Y. and Mary, T., Performance and scalability of the block low-rank multifrontal factorization on multicore architectures, ACM Transactions on Mathematical Software, Vol. 45, No. 1 (2019), Article 2, pp. 1-26.

Arai, K., Okada, H. and Yusa, Y., A new formulation of J-integral range $\Delta \mathrm{J}$ using three-dimensional equivalent domain integral for finite deformation elastic-plastic problem, Transactions of the JSME (in Japanese), Vol. 84, No. 867 (2018), Paper No. 18-00309, pp. 1-19.

Bathe, K. J. and Cimento, A. P., Some practical procedures for the solution of nonlinear finite element equations, Computer Methods in Applied Mechanics and Engineering, Vol. 22, No. 1 (1980), pp. 59-85.

CERFACS, CNRS, ENS Lyon, INP Toulouse, Inria, Mumps Technologies and University of Bordeaux, MUMPS: a parallel sparse direct solver (online) (1991-2021), <http://mumps.enseeiht.fr/>, (accessed on 5 January 2021).

Crisfield, M. A., A faster modified Newton-Raphson iteration, Computer Methods in Applied Mechanics and Engineering, Vol. 20, No. 3 (1979), pp. 267-278.

De Borst, R., Crisfield, M. A., Remmers, J. J. C. and Verhoosel, C. V., Nonlinear Finite Element Analysis of Solids and Structures, Second Edition (2012), John Wiley \& Sons.

De Souza Neto, E. A., Perić, D. and Owen, D. R. J., Computational Methods for Plasticity: Theory and Applications (2008), John Wiley \& Sons (translated into Japanese by Terada, K., Akiyama, M., Asai, M., Ishii, T., Oide, K., Okazawa, S., Kuboki, T., Kurumatani, M., Saiki, I., Takaki, T., Tsutsumi, S., Matsui, K., Yamakawa, Y. and Watan- 
abe, I. (2012), Morikita Publishing).

Gelin, J. C. and Picart, P., Use of quasi-Newton methods for large strain elastic-plastic finite element computations, Communications in Applied Numerical Methods, Vol. 4, No. 4 (1988), pp. 457-469.

Geradin, M., Idelsohn, S. and Hogge, M., Nonlinear structural dynamics via Newton and quasi-Newton methods, Nuclear Engineering and Design, Vol. 58, No. 3 (1980), pp. 339-348.

Geradin, M., Idelsohn, S. and Hogge, M., Computational strategies for the solution of large nonlinear problems via quasiNewton methods, Computers \& Structures, Vol. 13, No. 1 (1981), pp. 73-81.

Hisada, T. and Noguchi, H., Foundation and Application of Nonlinear Finite Element Method (1995), Maruzen Publishing (in Japanese).

Karypis, G. and Kumar, V., A fast and high quality multilevel scheme for partitioning irregular graphs, SIAM Journal on Scientific Computing, Vol. 20, No. 1 (1998), pp. 359-392.

Karypis, G., Family of graph and hypergraph partitioning software (online) (2006-2021), $<$ http://glaros.dtc.umn.edu/gkhome/views/metis>, (accessed on 5 January 2021).

Laursen, T. A. and Maker, B. N., An augmented Lagrangian quasi-Newton solver for constrained nonlinear finite element applications, International Journal for Numerical Methods in Engineering, Vol. 38, No. 21 (1995), pp. 3571-3590.

Matthies, H. and Strang, G., The solution of nonlinear finite element equations, International Journal for Numerical Methods in Engineering, Vol. 14, No. 11 (1979), pp. 1613-1626.

Mei, R., Li, C. and Liu, X., A NR-BFGS method for fast rigid-plastic FEM in strip rolling, Finite Elements in Analysis and Design, Vol. 61 (2012), pp. 44-49.

MDF Subcommittee, Atomic Energy Research Committee, The Japan Welding Engineering Society, Data base on fatigue and ductile fracture under multi-axial load (online) (2017), <http://www-it.jwes.or.jp/fatigue_db/>, (accessed on 5 January 2021).

Nayak, G. C. and Zienkiewicz, O. C., Note on the 'alpha'-constant stiffness method for the analysis of non-linear problems International Journal for Numerical Methods in Engineering, Vol. 4, No. 4 (1972), pp. 579-582.

Nocedal, J., Updating quasi-Newton matrices with limited storage, Mathematics of Computation, Vol. 35, No. 151 (1980), pp. 773-782.

Okada, H., Ishizaka, T., Takahashi, A., Arai, K. and Yusa, Y., 3D J-integral evaluation for solids undergoing large elasticplastic deformations with residual stresses and spatially varying mechanical properties of a material, Engineering Fracture Mechanics, Vol. 236 (2020), Paper No. 107212, pp. 1-27.

Shigeno, Y., Performance enhancement of FEM code for soils and foundations with thread parallelization, AIJ Journal of Technology and Design, Vol. 19, No. 41 (2013), pp. 345-350 (in Japanese).

Wissmann, J. W. and Hauck, C., Efficient elastic-plastic finite element analysis with higher order stress-point algorithms, Computers \& Structures, Vol. 17, No. 1 (1983), pp. 89-95.

Wriggers, P., Nonlinear Finite Element Methods (2008), Springer-Verlag.

Yoshimura, S., Shioya, R., Noguchi, H. and Miyamura, T., Advanced general-purpose computational mechanics system for large-scale analysis and design, Journal of Computational and Applied Mathematics, Vol. 149, No. 1 (2002), pp. 279-296.

Yusa, Y., Okada, H., Yamada, T. and Yoshimura, S., Scalable parallel elastic-plastic finite element analysis using a quasiNewton method with a balancing domain decomposition preconditioner, Computational Mechanics, Vol. 61, No. 6 (2018), pp. 1563-1581. 